\title{
The Design and Usability Testing of a Mobile Application to Aid in Child-to-Adult-Care Transition
}

\author{
Jeremy Dixon ${ }^{1}$, Josh Dehlinger ${ }^{1}$, and Shannan DeLany Dixon ${ }^{2}$ \\ ${ }^{1}$ Department of Computer and Information Sciences Towson University, Towson, MD \\ ${ }^{2}$ Department of Pediatrics University of Maryland - School of Medicine, Baltimore, MD \\ jdixon6@students.towson.edu, jdehlinger@towson.edu, \\ smdixonasom. umaryland.edu
}

\begin{abstract}
As mobile devices become more central in our lives, accessibility and utility for users becomes essential. The widespread availability of mobile devices introduces a number of challenges to traditional software engineering including: 1. mobile user interfaces differ from traditional interfaces; and, 2. the diversity of mobile platforms. As part of a larger research effort, this poster presents the design, implementation and initial testing of a mobile application aimed at helping the child-to-adult-care transition process for children with chronic disease. Based on recommendations from the American Academy of Pediatrics, the application will help guide patients through four main components of transition: 1 . assess transition readiness; 2 . plan the transition; 3 . implement the transition; and, 4. help document the transition. The design, implementation and testing of a mobile application may ease the transition process. By leveraging modern cross-compilation tools, this application can be implemented on multiple mobile platforms. This will lead to a variety of users, including those who may be differently-abled, to have a more fluid transition to their new health care providers.
\end{abstract}

Keywords: mobile applications, human-computer interaction, accessibility.

\section{Introduction}

As mobile application usage has grown, user accessibility and utility for those who are differently-abled has become essential [1]. A large portion of the differently-abled population must undergo process of medical transition. Medical transition is defined as the process of an individual taking complete control of their medical care and transferring care from a pediatric focused medical team to one based in adult medicine. The transition from pediatric care to adult care occurs most commonly in an individual when they are in their late teens or early twenties [2]. Transition is an important step in the healthcare of a young adult and a failed or incomplete transition may cause physical and/or mental health issues for adolescents with chronic conditions [3]. Medical transition for patients with chronic diseases from pediatricbased care to adult-based care is one that has been studied, developed and implemented for a number of years; recently, it has become a top priority in 
healthcare [2]. Due to the complexities of the transition process, a well-designed, intuitive mobile application should improve the standardization and ease of care for these patients. The significance of this applied research is to bring needed attention to the evaluation and necessity of a mobile application that assists with pediatric-to-adult transition which is considered to be a critical issue in healthcare today.

The application is designed to help with the transition process by leading the patient through the four main components recommended by the American Academy of Pediatrics [2]. First, the application will illustrate key questions to help decide if a patient is ready to initiate the transition process. These questions will help the patient to assess their knowledge of the disorder, their personal patient information, their ability to complete daily activities, and an assessment of communication practices. If a patient is not ready to start the transition process, the application will provide guidance as to what tasks need to be established prior to starting the transition.

Second, the application will help patients plan the transition process. It will provide suggestions for goals to complete along with realistic timelines. Goals include: understanding the importance of obtaining medical records, identifying current and future care team members, demonstrating the ability to communicate a working knowledge of the genetic disorder as well as places to identify disease based information and understanding the complexities of management of the disorder. The main purpose of this phase is to help educate a patient as to what is involved in a successful transition.

Third, the application will help initiate patient transition. The primary goal of the implementation phase is to educate all involved parties regarding the plan for transition. In many cases, this will require the patient, caregivers, family members and the healthcare team to be educated as to a patient's condition, the expectations of care, and the timeline for completion. The application will provide a list of resources, a task completion checklist and talking points to help with the implementation phase but will not provide any disease-specific resources. The talking point section will include lists of questions to ask current and future care providers, insurance companies, and pharmacies [2]. Additionally, a list of talking points will be provided to the patient within the mobile application to help initiate and engage their family and care providers in open and honest conversations.

Finally, the application will help document important aspects of the transition process, such as important insurance information, contact information for the healthcare team, and checklists to help ensure that nothing is missed [4]. The complete application will be able to be emailed or printed so that the patient may provide this information to their care providers to add to their medical record.

\section{Application Design}

Designing the application so that it meets the needs of the transition plan can be complex as each patient is different. As the goal is to complete the four transition plan components, it is important for the application to meet each of those needs. 
Additionally, the application will store important information about both the patient and their medical care team. Finally, the application needs to provide both assistance for the user interface and any required usability enhancements. The application is being built using the Sencha Touch 2 mobile framework. Importantly, Sencha Touch allows developers to create HTML5 based mobile apps that work on Android, iOS and Blackberry devices [4].

The first step is to assess transition readiness [2]. The transition readiness evaluations often look like a checklist or a series of questions. For example, a question might be, "I understand my health care needs, and disability and can explain my needs to others" [5]. If these questions are yes/no questions, Sencha Touch includes a list with a checkbox option. If the question requires options such as, "Yes", "I want to do this", "I need to learn how", or "Someone else will have to do this Who?" then a dropdown can be employed with a comment section. The comment section will provide detailed explanations of the task itself to help patients minimize any confusion. Figure 1 shows an example of the option boxes in Sencha.

The second phase involves planning the transition [2]. This phase includes developing a timeline for the transition, identifying responsibilities, and developing a skill list. From an application implementation standpoint, this phase has some of the components related to the previous phase with the exception that the list of caregivers will be documented. In order for the patient to record their current caregiver information, a series of forms were generated that document important contact information and comments regarding each person on the team. This will be very important when seeking new caregivers in the next phase. As in the previous phase, this section of the application will also contain drop down boxes, which will provide descriptions of the services that each type of physician provides. Figure 2 shows an example of the interface that will allow new information to be stored in the application. In this case, the information is patient related.

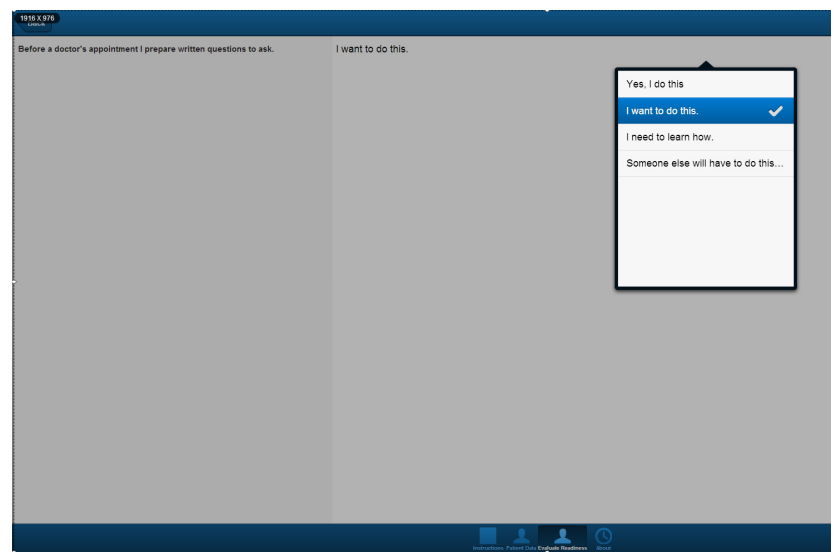

Fig. 1. Example of Option Boxes in Sencha (as viewed in Chrome) 


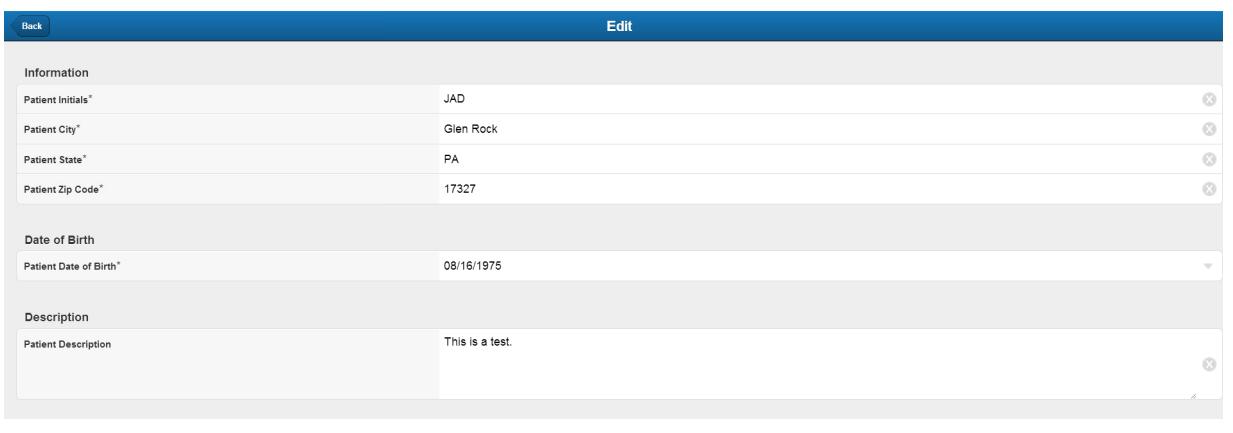

Fig. 2. Example of Editable Form in Sencha (as viewed in Chrome)

The third phase involves implementing the plan through education and goal achievement [2]. This phase will provide the user with a variety of goals related to the transition process. For example, in this phase, the user may be provided with a goal of scheduling an interview with a prospective doctor in order to discuss their diagnoses. The application will also provide an important topic list to discuss with the prospective doctor and how to make sure that they will be an appropriate fit for care of the patient. From an implementation standpoint, this phase requires checklists with a number of forms. The checklists and forms are generated from a thorough review of the literature and discussions with various transition clinics.

The fourth phase involves documenting the transition process. As the patient continues through the transition process, it is important to know what areas have been completed and what areas still need to be worked on to complete the process. This part of the application will review the information already reported and generate a summary of additional steps to be completed. From an application implementation standpoint, this phase will query to database to see what aspects of the transition have not been completed. It will list all of the sections that need to be worked on including links to the appropriate part of the application. In many ways, this is a dashboard that shows the user's progress through the transition.

\section{Conclusion}

In order to ensure the best transition experiences, it is important for this application to guide the patient in order to ensure that the care is uninterrupted and of high quality. The design, implementation and testing of a mobile application may significantly help with the transition process. By leveraging modern cross-compilation tools, the application can be implemented on multiple mobile operating systems so that a variety of users, including those who may be differently-abled, to have a fluid transition to their new health care providers. The application's success will be measured in three ways: 
- Users will successfully complete their transition in less than 6 months.

- Users will have no lapses in medical care during the transition process and for the six months after the transition has been completed.

- The application will receive generally positive reviews from both the patients and care givers.

Providing high quality healthcare to a person going through the transition from pediatric care to adult care can be a complex and time consuming effort. The best transition experiences are those where the care is uninterrupted and high quality and are patient driven. Currently, there are a variety of checklists and tools available to help people who are going through the transition period to complete the process however are not provided in a centralized location and have varying results.

Advances in medical knowledge and skills are helping children and young adults with chronic conditions live much longer than in previous generations. Due to this, the population of people going through the transition process is increasing. By designing a mobile application framework that helps with the transition process, the efforts required to successfully complete this may be improved. Once the application is developed and validated, it may be distributed to a broader patient base throughout the medical system to determine effectiveness to a global patient population.

Acknowledgements. We would like to acknowledge New York Mid Atlantic Consortium for Genetics and Newborn Screening Services (NYMAC) for their support and funding of this project.

\section{References}

1. Dehlinger, J., Dixon, J.: Mobile Application Software Engineering: Challenges and Research Directions. In: Mobile Software Engineering Workshop, MobiCase 2011, Santa Monica (2011)

2. American Academic of Pediatrics, Supporting the Transition from Adolescence to Adulthood in the Medical Home. Pediatrics, 182-200 (2011)

3. Binks, J.A., Barden, W.S., Burke, T.A., Young, N.L.: What Do We Really Know About the Transition to Adult-Centered Health Care? A Focus on Cerebral Palsy and Spina Bifida. Archives of Physical Medicine Rehabilitation 88, 1064-1073 (2007)

4. Sencha Inc., Sencha Touch (Feburary 2013), http: / /www. sencha.com/products/touch/

5. New England Consortium of Metabolic Programs, Transition Toolkit (August 2010), http://newenglandconsortium.org/for-families/transition-toolkit/ 